Research Paper

\title{
Cisplatin Nephrotoxicity Might Have a Sex Difference. An analysis Based on Women's Sex Hormone Changes
}

\author{
Wei-Yu Chen ${ }^{1^{*}}$, Ching-Hsing Hsiao ${ }^{*}$, Yi-Chen Chen ${ }^{3}$, Chung-Han Ho ${ }^{3,4,5}$, Jhi-Joung Wang6, Chung-Hsi \\ Hsing ${ }^{7,8}$, Hsien-Yi Wang ${ }^{9,10}$, Wei-Chih Kan ${ }^{9,11}$, Chia-Chun $\mathrm{Wu}^{4,9}$ \\ 1. Department of Hematology and Oncology, Chi Mei Medical Center, Yongkang District, Tainan City 710, Taiwan. \\ 2. Department of Ophthalmology, Chi Mei Hospital, Jiali District, Tainan, Taiwan. \\ 3. Department of Medical Research, Chi Mei Medical Center, Yongkang District, Tainan City 710, Taiwan. \\ 4. Department of Pharmacy, Chia Nan University of Pharmacy and Science, Rende District, Tainan, Taiwan. \\ 5. Department of Hospital and Health Care Administration, Chia Nan University of Pharmacy and Science, Tainan, Taiwan. \\ 6. Department of Medical Research, Chi Mei Medical Center, Yongkang District, Tainan City 710, Taiwan. \\ 7. Department of Anesthesiology, Chi Mei Medical Center, Yongkang District, Tainan City 710, Taiwan. \\ 8. Department of Anesthesiology, College of Medicine, Taipei Medical University, Taipei 11031, Taiwan. \\ 9. Department of Nephrology, Department of Internal medicine, Chi Mei Medical Center, Yongkang District, Tainan City 710, Taiwan. \\ 10. Department of Sports Management, College of Leisure and Recreation Management, Chia Nan University of Pharmacy and Science, Rende District, Tainan, \\ Taiwan. \\ 11. Department of Biological Science and Technology, Chung Hwa University of Medical Technology, Rende District, Tainan City 717, Taiwan. \\ * Wei-Yu Chen and Ching-Hsing Hsiao contributed equally to the writing of this work. \\ $\square$ Corresponding author: Chia-Chun Wu, MD, Department of Nephrology, Chi Mei Medical Center, Taiwan, 901 Zhonghua Road, Yongkang District, Tainan \\ City 710, Taiwan. Tel: +886-6-281-2811 ext. 52639; Fax: +886-6-282-8928; E-mail: chiachun4481@gmail.com \\ (C) Ivyspring International Publisher. This is an open access article distributed under the terms of the Creative Commons Attribution (CC BY-NC) license \\ (https://creativecommons.org/licenses/by-nc/4.0/). See http://ivyspring.com/terms for full terms and conditions.
}

Received: 2017.03.15; Accepted: 2017.09.20; Published: 2017.10.23

\begin{abstract}
Background: A sex difference in cisplatin-induced nephrotoxicity $(\mathrm{CIN})$ has been reported in human and animal studies. We examined in humans whether it is associated with sex-hormone changes.

Methods: In this retrospective nationwide cohort study, we used Taiwan's National Health Insurance Research Database (NHIRD) to identify patients with a history of malignancy and cisplatin treatment. Patients diagnosed with kidney disease before cisplatin treatment and those with sex-organ malignancies were excluded. A diagnosis of kidney disease within 90 days after the first administration of cisplatin was the study outcome. Risk factors were estimated using a Cox regression model. Subgroup analyses were performed based on different women's estrogen levels in phases of childbearing, perimenopause, and postmenopause.

Results: A retrospective analysis of the records of 3973 men (mean age: $56.15 \pm 12.85$ years) and 1154 women (mean age: $56.31 \pm 12.40$ years) showed that 1468 (36.95\%) men and 451 (39.08\%) women had a new diagnosis of kidney disease. The risk factors were being $>55$ years old, a high comorbidity score, and a history of aminoglycoside treatment. Only postmenopausal women had a significantly higher risk of kidney injury (hazard ratio: 1.28; $95 \% \mathrm{Cl}$ : 1.02-1.61) than did men.

Conclusions: Perimenopausal women have a significantly higher risk of $\mathrm{CIN}$ than do men, which might be explained by women's higher levels of estrogen. Additional studies on the underlying mechanisms of the sex difference of CIN are needed.
\end{abstract}

Key words: cisplatin, nephrotoxicity, sex difference, chronic kidney disease, acute kidney disease

\section{Introduction}

Sex-based differences are reported in various diseases, including acute kidney injury (AKI) and chronic kidney disease (CKD) [1-7].
In general, women seem to have less frequent and less severe AKI and CKD than do men [2,5,8]. Some animal studies [8-11] report that females are 
more resistant to ischemic AKI. Sex hormones are usually considered at least partially responsible for the sex difference in disease expression, and estradiol in particular is considered a protective hormone in AKI [2].

Cisplatin is a platinum-based chemotherapeutic agent widely used for solid tumors in lung, head and neck, ovary, and urinary bladder cancer. It kills cancer cells by crosslinking the DNA base, which induces DNA damage and impairs DNA repair. However, cisplatin has well-known side effects - neurotoxicity, nephrotoxicity, and bone marrow suppressionwhich often limits its clinical use [12]. Cisplatin usually induces AKI and is believed to contribute to the development of CKD [13-15]. The mechanisms are complicated and involve proximal tubule injury, inflammation, oxidative stress, and vascular injury, but they are not completely understood [16]. In proximal tubule injury, the organic cation transporters 2 (OCT2), which are primarily in the basolateral membrane of the renal proximal tubules, are considered important mediators of cisplatin uptake and a cause of renal tubular cell injury.

Although women seem to have less frequent and less severe AKI and CKD than do men, some studies $[15,19]$ report that being female is a risk factor of CIN. In animal-model CIN studies [20-26], the benefits of renal protective drugs were detected in male but not in female rats, and treatment with estradiol did not protect the kidney but exacerbated the CIN. There are three life stages based on the ovary function and sex hormone changes in women: childbearing, perimenopause, and menopause [27]. The perimenopause stage is the period immediately before and one year after menopause, usually between 45-55 years old. In the perimenopause stage, estrogen levels are high and fluctuating, but they decline in the menopause stage [28].

Our review of the literature revealed that no published clinical study focuses on whether the sex difference in CIN is associated with sex hormone changes. We compared the development of CIN between men and women based on the three life stages of women.

\section{Materials and Methods}

\section{Data source}

We did a retrospective cohort study using the Longitudinal Health Insurance Database 2000 (LHID2000) (http://nhird.nhri.org.tw/en/Data_ Subsets.html) subset of the Taiwan National Health Insurance Research Database (NHIRD) provided by the Bureau of National Health Insurance, Department of Health. Because the personal information of all patients is encrypted to avoid violating their privacy rights, the institutional review board of Chi Mei Medical Center waived the requirement for signed informed consent (IRB No. 10508-E02).

\section{Patients}

We extracted the data of patients with an ICD-9-CM diagnostic code of 140-208 and with a catastrophic illness certification: the certification makes the malignancy diagnosis more reliable. All patients in the LHID2000 treated with cisplatin were included. To ensure that all who had been treated with cisplatin did not have comorbid CKD, any patients with a diagnosis of CKD (ICD-9-CM: 042, 223,403, 404, 581, 583, 585, 586, 587, 588, 589, 593, 753, 591, 016.0, 095.4, 236.9, 250.4, 271.4, 274.1, 440.1, 442.1, $447.3,446.21)$ before being treated with cisplatin were excluded. Patients younger than 20 years old, and those with sex organ malignancies (ICD-9-CM: $179,180,182,183,184,185, \quad 186,187,189,199,198.0,198.1)$, female-predominant breast cancer (ICD-9-CM: 174), or more than one kind of malignancy were also excluded. Patients with missing birthdate or sex information were also excluded. Nonsteroidal anti-inflammatory drugs (NSAIDS) are often prescribed before cisplatin treatment, nephrotoxic contrast media are used for computed tomography (CT), and, after cisplatin treatment, aminoglycosides (e.g., gentamicin and amikacin) are often prescribed for infection control. All such nephrotoxic substances are considered confounding factors. The Charlson comorbidity index (CCI) (without index values from the tumor) was used to evaluate comorbidities, if the comorbidity code appeared at least once in the admission code or three times in the Outpatient Department diagnosis codes within one year before cisplatin treatment. In addition, to evaluate the effect of estrogen in women, they were analyzed in agestratified subgroups: childbearing, perimenopause, and postmenopause.

\section{Outcome Variables}

The outcome of interest was the development of acute or chronic kidney injury with or without dialysis. The ICD-9-CM codes for these outcomes are listed in Table 1. Patients were determined to be on hemodialysis or peritoneal dialysis based on its ICD-9-CM code. All patients who did not have an outcome of interest or who died within 90 days were right censored, or else they were followed-up until the date they were diagnosed with any of the outcomes of interest at least once in outpatient and in inpatient records. 
Table 1. ICD-9-CM codes for acute kidney injury (AKI) and chronic kidney disease (CKD)

ICD-9-CM code

584.9 acute kidney injury (AKI)

580-589 chronic kidney disease (CKD: nephritis, nephrotic syndrome, and nephrosis)

753 (congenital anomalies of urinary system)

403 (hypertensive chronic kidney disease)

404 (hypertensive heart and chronic kidney disease)

250.4 (diabetes with renal manifestations)

274.1 (gouty nephropathy)

440.1 (atherosclerosis of renal artery)

442.1 (other aneurysm of renal artery)

447.3 (hyperplasia of renal artery)

572.4 (hepatorenal syndrome)

642.1 (hypertension secondary to renal disease, complicating pregnancy,

childbirth, and the puerperium)

646.2 (unspecified renal disease in pregnancy, without mention of hypertension)

\section{Statistical Analysis}

The mean difference between men and women for continuous variables - age, CCI score, and number of NSAID prescriptions-was analyzed using Student's t test. For categorical variables - age group, comorbidities, NSAID prescriptions, aminoglycoside treatment, exposure to contrast medium, CKD, and AKI-Pearson's $\chi^{2}$ test was used to estimate the distribution difference between men and women. Kaplan-Meier curves were plotted to compare the incidence of kidney injury between men and women during the study period, and the log-rank test was used to examine the significance of the difference. Cox proportional hazard regression models adjusted for all potential confounding variables were also used. Because age and different cancer types treated with cisplatin are associated with different risks of kidney injury, we used a stratified analysis for different age

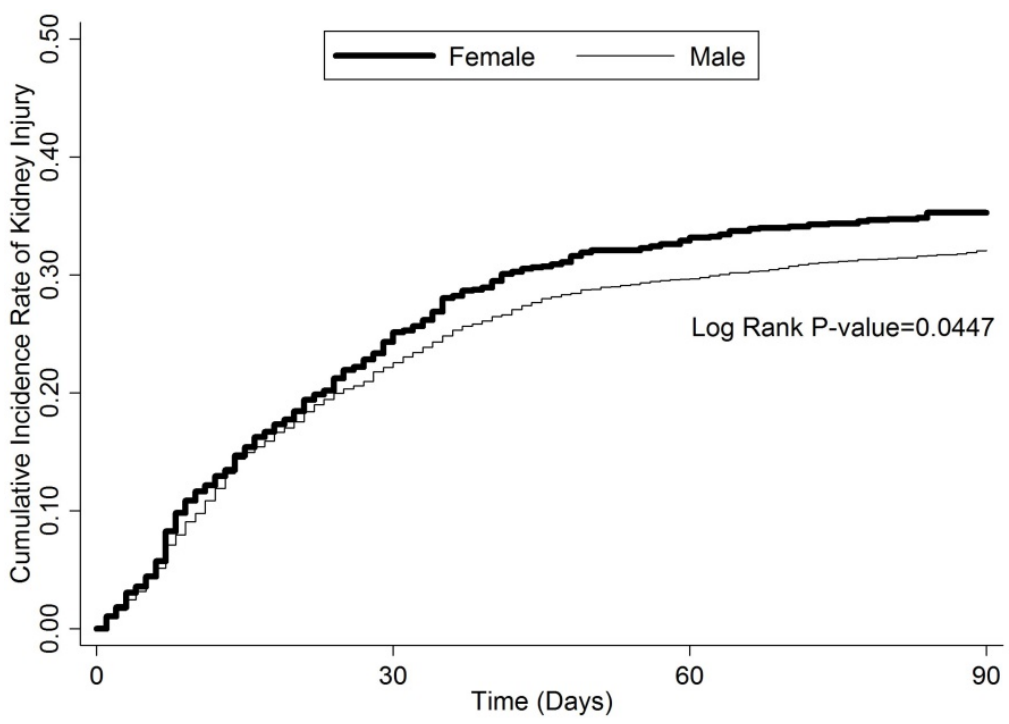

Figure 1. The cumulative incidence rate of kidney injury within 90 days after first cisplatin administration. groups and cancer types. SAS 9.4 for Windows (SAS Institute, Inc., Cary, NC) was used for all statistical analyses. Kaplan-Meier curves were generated using STATA 12 for Windows (Stata Corp., College Station, TX). Significance was set at $\alpha=0.05$.

\section{Results}

We identified, in the LHID2000, 53,731 patients with cancer; the birthdates and sex of all were indicated. However, 81 patients $<20$ years old were excluded. Patients with more than one type of cancer or with malignant sex organs $(14.8 \%)$ were also excluded. There were 5127 patients (3973 men [mean age: $56.15 \pm 12.85$ years]; 1154 women [mean age: $56.31 \pm 12.40$ years]) without previously diagnosed kidney disease who had been treated with cisplatin for cancer. The mean follow-up time was $61.16 \pm$ 242.02 days with no significant sex-based difference $(P$ $=0.9$ ). Women had higher CCI scores, more NSAID prescriptions, and fewer aminoglycoside treatments than did men. There was no significant sex-based difference in exposure to contrast medium (Table 2).

Overall, 451 (39.08\%) women and 1468 (36.95\%) men had newly diagnosed AKI (9 women; 37 men), CKD (445 women; 1439 men), or both (3 women; 8 men) within 90 days after cisplatin treatment (nonsignificant difference) (Table 2). A Kaplan-Meier survival analysis showed that women had a significantly higher risk of developing $\operatorname{CIN}(P=$ 0.0457) (Figure 1). A Cox regression proportional hazards analysis showed that being $>55$ years old, having a CCI $>2$, and having a history of aminoglycoside treatment after cisplatin therapy were risk factors, but that having diabetes mellitus was protective against developing CIN. Being female, which initially was a risk factor for developing CIN, became nonsignificant after the analysis had been adjusted for hypertension (HTN), CCI score, number of NSAID prescriptions, and exposure to contrast medium or aminoglycoside after cisplatin treatment (Table 3). However, if we stratified patients by age group (childbearing [< 45 years], perimenopausal [45-55 years], and postmenopausal [> 55 years $]$ ), perimenopausal women had a risk of developing CIN 1.28 times greater than did men of the same age $(P=0.0333)$ (Table 4, Figure 2). A subgroup analysis by cancer type showed that women with head and neck rather than gastrointestinal tract or lung cancer had a higher risk of kidney injury after cisplatin treatment (Table 5). 
(A) Age group (20-45)

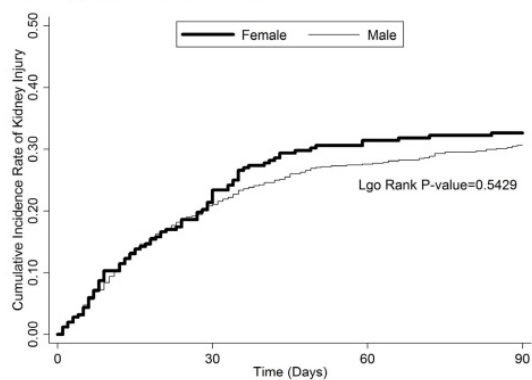

(B) Age group (45-55)

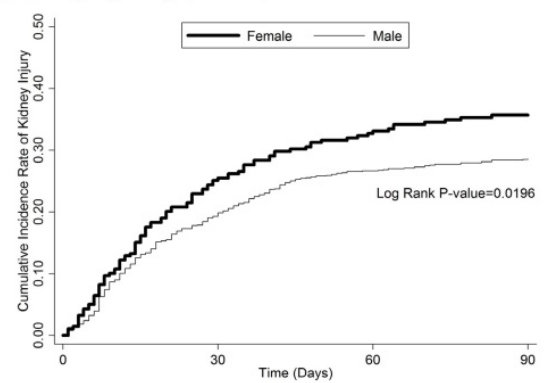

(C) Age group (> 55)

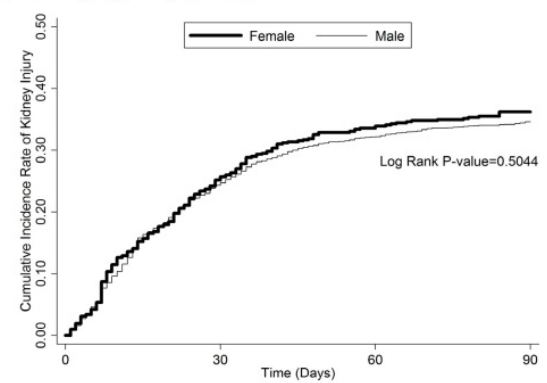

Figure 2. The cumulative incidence rate of kidney injury within 90 days after first cisplatin administration in different life stage of women: (A) childbearing stage 20-45 years old (B) perimenopause stage 45-55 years old (C) postmenopause stage (>55 years old) This figure had been presented at ASN Kidney Week 2016 in Chicago, IL. More information can be found at www.asn-online.org.

\section{Discussion}

We found a sex difference in CIN: women, especially perimenopausal women (45-55 years old), have a higher risk of developing CIN than do men. Being $>55$ years old and exposed to aminoglycoside after cisplatin treatment are risk factors for developing CIN.

Table 2. Demographic analysis of cisplatin-induced nephrotoxicity patients stratified by gender.

\begin{tabular}{|c|c|c|c|}
\hline \multirow[t]{2}{*}{ Variables } & \multicolumn{2}{|c|}{ Gender } & \multirow[t]{2}{*}{$P$ value } \\
\hline & Female $(n=1154)$ & Male $(n=3973)$ & \\
\hline Age (years), mean $\pm S D$ & $56.31 \pm 12.40$ & $56.15 \pm 12.85$ & 0.7026 \\
\hline \multicolumn{4}{|l|}{ Age group, $\mathrm{n}(\%)$} \\
\hline $20-45$ (years) & $254(22.01)$ & $800(20.14)$ & $0.0089^{*}$ \\
\hline 45-55 (years) & $280(24.26)$ & 1145 (28.82) & \\
\hline > 55 (years) & $620(53.73)$ & $2028(51.04)$ & \\
\hline \multicolumn{4}{|l|}{ Comorbidities } \\
\hline Diabetes mellitus & $150(13.00)$ & $447(11.25)$ & 0.1033 \\
\hline Hypertension & $324(28.08)$ & $894(22.50)$ & $<0.0001^{*}$ \\
\hline \multicolumn{4}{|l|}{ CCI score } \\
\hline mean $\pm S D$ & $4.05 \pm 3.10$ & $3.69 \pm 3.15$ & $0.0008^{*}$ \\
\hline 0 & $290(25.13)$ & $1144(28.79)$ & $0.0034^{*}$ \\
\hline $1-2$ & $185(16.03)$ & 714 (17.97) & \\
\hline$>2$ & $679(58.84)$ & $2115(53.23)$ & \\
\hline \multicolumn{4}{|l|}{ NSAIDs prescriptions (n) } \\
\hline$($ mean $\pm S D)$ & $32.55 \pm 38.67$ & $22.74 \pm 29.24$ & $<0.0001^{*}$ \\
\hline$<25$ & $640(55.46)$ & $2810(70.73)$ & $<0.0001^{*}$ \\
\hline$\geq 25$ & $514(44.54)$ & $1163(29.27)$ & \\
\hline \multicolumn{4}{|l|}{ Contrast medium } \\
\hline Yes & $745(64.56)$ & $2685(67.58)$ & 0.0547 \\
\hline No & 409 (35.44) & $1288(32.42)$ & \\
\hline \multicolumn{4}{|l|}{ Gentamicin and Amikacin } \\
\hline Yes & 209 (18.11) & $874(22.00)$ & $0.0044^{*}$ \\
\hline No & 945 (81.89) & $3099(78.00)$ & \\
\hline \multicolumn{4}{|l|}{ CKD or AKI } \\
\hline Yes & 451 (39.08) & 1468 (36.95) & 0.1877 \\
\hline No & 703 (60.92) & 2505 (63.05) & \\
\hline
\end{tabular}

This is the first database study to focus on the sex difference in CIN based on sex hormone changes in women. Our finding is basically consistent with the findings of two studies [15, 19] which reported that renal function declined more in women than in men after cisplatin treatment. We used a short ( 3 months) instead of a long-term (6 years) follow-up [15] to avoid the bias from other complicated conditions that easily injure the kidney and limit the life span of cancer patients. Additionally, patients with ovarian cancer were included in one of those studies [19] but excluded in ours. In contrast, another study [14] found no sex difference in its observational clinical study of CIN. Perhaps the difference in the ethnicity of its study population (adults in Detroit, Michigan, USA), high percentage of male patients, and their lack of age stratification led to different results.

Sex steroids (estradiol, progesterone, and testosterone) are considered pivotal to the sex difference in renal disease. Animal studies report that estradiol provides major renoprotection because of its antioxidant, and vasodilation, anti-apoptosis, and anti-inflammation functions. The role of progesterone in renal disease is less clear, but it can be renoprotective or harmful under different conditions. Testosterone seems to have pro-oxidant and vasoconstriction functions that mediate renal injury [29]. The same group conducted a series of studies on the sex difference and the possible role of estradiol in $\mathrm{CIN}$ in rats and found that L-arginine and losartan and erythropoietin protected male but not female rats from CIN, $[20,21,25,26]$ and that some of these substances were harmful to female rats [20, 21]. Furthermore, they showed that the physiological dose of estradiol abolished the renoprotection of erythropoietin, and that the pharmacological dose of estradiol abolished the renoprotection of Vitamin C, Vitamin E, and losartan in bilaterally ovariectomized rats [22-24]; estradiol-upregulated oxidative stress was suggested as the mechanism [23]. A recent study [30] reported that combined estrogen and progesterone treatment was dose-dependently renoprotective against CIN in bilaterally ovariectomized female rats. The study showed that an 
intramuscular injection of progesterone $(10 \mathrm{mg} / \mathrm{kg})$ plus estradiol $(0.5 \mathrm{mg} / \mathrm{kg})$ intramuscular injection protected the kidney more than did progesterone alone, but when the same dose of progesterone was combined with a higher dose of estradiol $(1 \mathrm{mg} / \mathrm{kg})$, the kidney injury score and kidney oxidase stress marker paradoxically increased. This interesting finding was consistent with our study result that a higher estradiol level in perimenopausal women indicated a higher risk of developing CIN.

Table 3. Cox proportional hazards model analysis for risk of developing kidney injury

\begin{tabular}{|c|c|c|c|c|}
\hline Variables & $\begin{array}{l}\text { Crude HR } \\
(95 \% \mathrm{CI})\end{array}$ & $P$ & $\begin{array}{l}\text { Adjusteda HR } \\
(95 \% \mathrm{CI})\end{array}$ & $P$ \\
\hline \multicolumn{5}{|l|}{ Sex } \\
\hline Female & $\begin{array}{l}1.12 \\
(1.00-1.25)\end{array}$ & $0.0457^{*}$ & $\begin{array}{l}1.12 \\
(1.00-1.25)\end{array}$ & 0.0524 \\
\hline Male & 1.00 & & 1.00 & \\
\hline \multicolumn{5}{|l|}{ Age } \\
\hline $20-45$ (years) & 1.00 & & 1.00 & \\
\hline $45-55$ (years) & $\begin{array}{l}1.00 \\
(0.83-1.11)\end{array}$ & 0.5747 & $\begin{array}{l}0.97 \\
(0.84-1.12)\end{array}$ & 0.663 \\
\hline$>55$ (years) & $\begin{array}{l}1.16 \\
(1.02-1.32)\end{array}$ & $0.0228^{*}$ & $\begin{array}{l}1.18 \\
(1.03-1.35)\end{array}$ & $0.0170^{*}$ \\
\hline $\begin{array}{l}\text { Diabetes mellitus } \\
\text { (DM) }\end{array}$ & $\begin{array}{l}0.87 \\
(0.75-1.02)\end{array}$ & 0.0943 & $\begin{array}{l}0.81 \\
(0.69-0.96)\end{array}$ & $0.0137^{*}$ \\
\hline Hypertension (HTN) & $\begin{array}{l}0.98 \\
(0.88-1.10)\end{array}$ & 0.7407 & $\begin{array}{l}0.93 \\
(0.82-1.05)\end{array}$ & 0.2543 \\
\hline \multicolumn{5}{|c|}{$\begin{array}{l}\text { Charlson comorbidity index (CCI) } \\
\text { score }\end{array}$} \\
\hline 0 & 1.00 & & 1.00 & \\
\hline $1-2$ & $\begin{array}{l}1.15 \\
(0.99-1.33)\end{array}$ & 0.0755 & $\begin{array}{l}1.14 \\
(0.97-1.33)\end{array}$ & 0.1019 \\
\hline$>2$ & $\begin{array}{l}1.20 \\
(1.07-1.35)\end{array}$ & $0.0019^{*}$ & $\begin{array}{l}1.16 \\
(1.03-1.31)\end{array}$ & $0.0131^{*}$ \\
\hline \multicolumn{5}{|c|}{ NSAIDs (number of prescriptions) } \\
\hline$<25$ & 1.00 & & 100.00 & \\
\hline$\geq 25$ & $\begin{array}{l}1.09 \\
(0.98-1.20)\end{array}$ & 0.1128 & $\begin{array}{l}1.08 \\
(0.98-1.20)\end{array}$ & 0.1315 \\
\hline \multicolumn{5}{|l|}{ Contrast medium } \\
\hline Yes & $\begin{array}{l}1.07 \\
(0.96-1.19)\end{array}$ & 0.2063 & $\begin{array}{l}1.03 \\
(0.93-1.14)\end{array}$ & 0.5791 \\
\hline No & 1.00 & & 1.00 & \\
\hline \multicolumn{5}{|c|}{ Gentamicin and Amikacin } \\
\hline Yes & $\begin{array}{l}1.36 \\
(1.21-1.51)\end{array}$ & $<0.0001^{*}$ & $\begin{array}{l}1.37 \\
(1.22-1.53)\end{array}$ & $<0.0001^{*}$ \\
\hline No & 1.00 & & 1.00 & \\
\hline \multicolumn{5}{|c|}{$\begin{array}{l}\text { Abbreviations: NSAIDs }=\text { nonsteroidal anti-inflammatory drugs; } \mathrm{HR}:=\text { hazard } \\
\text { ratio; } \mathrm{CI}=\text { confidence interval. } \\
{ }^{*} P<0.05 \text {. }\end{array}$} \\
\hline
\end{tabular}

The expression of OCT2 on proximal renal tubules is attributable to CIN. Studies [31,32] have reported that male rats express more OCT2 in proximal renal tubules than do female rats, and that the difference is related to testosterone. Human OCT2 critically mediates cisplatin nephrotoxicity. Sex differences in renal circulation might explain why CIN kidney damage is more severe in male than in female rats [33]. However, one study of OCT2 expression in the human kidney [34] did not show a significant difference between men and women, which might explain why our finding was not consistent with that of the animal model.

Table 4. Cox proportional hazards model analysis for risk of developing kidney injury (stratified by age group)

\begin{tabular}{|c|c|c|c|}
\hline Age group & Kidney injury n (\%) & Adjusteda HR (95\% CI) & $P$ \\
\hline \multicolumn{4}{|l|}{$20-45$ (years) } \\
\hline \multicolumn{4}{|l|}{ Sex } \\
\hline Female & $92(36.22)$ & $1.11(0.86-1.44)$ & 0.4043 \\
\hline Male & $287(35.88)$ & 1.00 & \\
\hline \multicolumn{4}{|l|}{ 45-55 (years) } \\
\hline \multicolumn{4}{|l|}{ Sex } \\
\hline Female & $109(38.93)$ & $1.28(1.02-1.61)$ & $0.0333^{*}$ \\
\hline $\begin{array}{l}\text { Male } \\
>55 \text { (years) }\end{array}$ & $393(34.32)$ & 1.00 & \\
\hline \multicolumn{4}{|l|}{ Sex } \\
\hline Female & $250(40.32)$ & $1.05(0.90-1.22)$ & 0.5549 \\
\hline Male & $788(38.86)$ & 1.00 & \\
\hline
\end{tabular}

Table 5. Hazard ratios of females and males with different cancer types (age: 45-55)

\begin{tabular}{|c|c|c|c|}
\hline Cancer type & $\mathrm{n}(\%)$ & Adjusteda HR (95\% CI) & $P$ \\
\hline All & $1425(100.00)$ & $1.28(1.02-1.61)$ & $0.0333^{*}$ \\
\hline Head and neck (140-149) & $650(45.61)$ & $1.50(1.02-2.20)$ & $0.0374^{*}$ \\
\hline GI tract (150-159) & 359 (25.19) & $1.27(0.88-1.84)$ & 0.196 \\
\hline Lung (160-165) & $342(24.00)$ & $1.00(0.76-1.31)$ & 0.974 \\
\hline Others & $74(5.19)$ & $1.03(0.52-2.05)$ & 0.9335 \\
\hline
\end{tabular}

The finding of our significant sex difference in CIN in perimenopausal patients might be related to high estradiol levels in this stage. We found that diabetes mellitus protected against developing CIN, which was compatible with findings in some animal studies [35-37], but the mechanisms remain unclear.

Our study has some limitations. First, we lack real laboratory data that specify AKI severity or the CKD stage and hormone levels: this information is not provided by the NHIRD. Second, the diagnosis codes might be misrecorded or over-recorded. Third, we have no information about cancer stage, nutritional status, or other conditions that make patients more susceptible to renal injury (not provided by the NHIRD). Fourth, although we have information about how much money was spent on prescribed cisplatin, we do not know the real dose administered to patients (not provided by the NHIRD). Fifth, nor do we know any details about CIN prophylactic therapy provided each patient (not provided by the NHIRD). Sixth, most of our study population is Han Chinese in Taiwan; therefore, the results might have an ethnicity-specific bias. Seventh, some female patients 
were taking hormone replacement therapy, which might have interfered with the results.

We conclude that physicians should pay more attention to CIN in female patients with cancer. Additional studies to identify the underlying mechanisms of the sex difference in CIN and the role of estrogen might help diminish the risk of women developing CIN.

\section{Acknowledgement}

This study was supported by grant CMFHR 10533 from Chi-Mei Medical Center.

\section{Competing Interests}

The authors have declared that no competing interest exists.

\section{References}

1. Malboosbaf R, Hosseinpanah F, Mojarrad M, Jambarsang S, Azizi F. Relationship between goiter and gender: a systematic review and meta-analysis. Endocrine. 2013; 43(3): 539-47.

2. Neugarten J, Golestaneh L. Gender and the prevalence and progression of renal disease. Adv Chronic Kidney Dis. 2013; 20(5): 390-5.

3. Ngo S, Steyn F, McCombe P. Gender differences in autoimmune disease. Front Neuroendocrinol. 2014; 35(3): 347-69.

4. Savic I. Sex differences in human epilepsy. Exp Neurol. 2014; 259: 38-43.

5. Grams ME, Sang Y, Ballew SH, et al. A meta-analysis of the association of estimated GFR, albuminuria, age, race, and sex with acute kidney injury. Am J Kidney Dis. 2015; 66(4): 591-601.

6. Regensteiner JG, Golden S, Huebschmann AG, Barrett-Connor E, Chang AY, Chyun D, et al. Sex Differences in the cardiovascular consequences of diabetes mellitus. Circulation. 2015; 132(25): 2424-47.

7. Tehrani S. Gender difference in the pathophysiology and treatment of glaucoma. Curr Eye Res. 2015; 40(2): 191-200.

8. Wei Q, Wang M-H, Dong Z. Differential gender differences in ischemic and nephrotoxic acute renal failure. Am J Nephrol. 2005; 25(5): 491-9.

9. Park KM, Kim JI, Ahn Y, et al. Testosterone is responsible for enhanced susceptibility of males to ischemic renal injury. J Biol Chem. 2004; 279(50): 52282-92.

10. Hodeify R, Megyesi J, Tarcsafalvi A, Mustafa HI, Seng NSHL, Price PM. Gender differences control the susceptibility to ER stress-induced acute kidney injury. Am J Physiol Renal Physiol. 2013; 304(7): F875-82.

11. Tanaka R, Tsutsui H, Ohkita M, Takaoka M, Yukimura T, Matsumura Y. Sex differences in ischemia/reperfusion-induced acute kidney injury are dependent on the renal sympathetic nervous system. Eur J Pharmacol. 2013; 714(1-3): 397-404.

12. Calvert H, Judson I, Van der Vijgh W. Platinum complexes in cancer medicine: pharmacokinetics and pharmacodynamics in relation to toxicity and therapeutic activity. Cancer Surv. 1993; 17: 189-217.

13. Jakob S, Arnold W, Marti H. Progressive renal failure after cisplatin therapy. Nephrol Dial Transplant. 1996; 11(2): 370-3.

14. Bhat ZY, Cadnapaphornchai $\mathrm{P}$, Ginsburg $\mathrm{K}$, et al. Understanding the risk factors and long-term consequences of cisplatin-associated acute kidney injury: an observational cohort study. PloS One. 2015; 10: e0142225.

15. Latcha S, Jaimes EA, Patil S, et al. Long-Term renal outcomes after cisplatin treatment. Clin J Am Soc Nephrol. 2016; 11(7): 1173-9.

16. Ozkok A, Edelstein CL. Pathophysiology of cisplatin-induced acute kidney injury. Biomed Res Int. 2014; 2014: 967826.

17. Ludwig T, Riethmuller C, Gekle $\mathrm{M}$, et al. Nephrotoxicity of platinum complexes is related to basolateral organic cation transport. Kidney Int. 2004; 66: $196-202$

18. Ciarimboli G, Ludwig T, Lang D, et al. Cisplatin nephrotoxicity is critically mediated via the human organic cation transporter 2. Am J Pathol. 2005; 167: 1477-84.

19. De Jongh F, Van Veen $\mathrm{R}$, Veltman $\mathrm{S}$, et al. Weekly high-dose cisplatin is a feasible treatment option: analysis on prognostic factors for toxicity in 400 patients. Br J Cancer. 2003; 88(8): 1199-206.

20. Haghighi $\mathrm{M}$, Nematbakhsh $\mathrm{M}$, Talebi $\mathrm{A}$, et al. The role of angiotensin II receptor 1 (AT1) blockade in cisplatin-induced nephrotoxicity in rats: gender-related differences. Ren Fail. 2012; 34(8): 1046-51.

21. Eshraghi-Jazi F, Nematbakhsh $\mathrm{M}, \mathrm{Nasri} \mathrm{H}$, et al. The protective role of endogenous nitric oxide donor (L-arginine) in cisplatin-induced nephrotoxicity: Gender related differences in rat model. J Res Med Sci. 2011; 16(11): 1389-96
22. Pezeshki $\mathrm{Z}$, Nematbakhsh $\mathrm{M}$, Nasri $\mathrm{H}$, et al. Evidence against protective role of sex hormone estrogen in cisplatin-induced nephrotoxicity in ovarectomized rat model. Toxicol Int. 2013; 20(1): 43-7.

23. Nematbakhsh M, Pezeshki Z, Eshraghi-Jazi F, et al. Vitamin E, vitamin C, or losartan is not nephroprotectant against cisplatin-induced nephrotoxicity in presence of estrogen in ovariectomized rat model. Int J Nephrol. 2012; 2012: 284896.

24. Pezeshki Z, Nematbakhsh M, Mazaheri S, et al. Estrogen abolishes protective effect of erythropoietin against cisplatin-induced nephrotoxicity in ovariectomized rats. ISRN Oncol. 2012; 2012.

25. Eshraghi-Jazi F, Nematbakhsh M, Pezeshki Z, et al. Sex differences in protective effect of recombinant human erythropoietin against cisplatin-induced nephrotoxicity in rats. Iran J Kidney Dis. 2013;7:383-9.

26. EL-Arabey AA. Re:Sex differences in nephro-protective effect of recombinant human erythropoietin in rats. Iran J Kidney Dis. 2015; 9(2):163.

27. World Health Organization. Research on the menopause in the 1990s (Report of a WHO scientific group, WHO Technical Report Series, 886). Geneva: World Health Organization. 1996

28. Prior JC. Perimenopause: the complex endocrinology of the menopausal transition. Endocr Rev. 1998; 19(4): 397-428.

29. Yanes LL, Sartori-Valinotti JC, Reckelhoff JF. Sex steroids and renal disease.: lessons from animal studies. Hypertension. 2008; 51(4): 976-81.

30. Ghasemi M, Nematbakhsh M, Pezeshki Z, et al. Nephroprotective effect of estrogen and progesterone combination on cisplatin-induced nephrotoxicity in ovariectomized female rats. Indian J Nephrol. 2016; 26(3): 167-75.

31. Yonezawa A, Masuda S, Nishihara K, et al. Association between tubular toxicity of cisplatin and expression of organic cation transporter rOCT2 (Slc22a2) in the rat. Biochem Pharmacol. 2005; 70(12): 1823-31.

32. Urakami $\mathrm{Y}$, Okuda $\mathrm{M}$, Saito $\mathrm{H}$, et al. Hormonal regulation of organic cation transporter OCT2 expression in rat kidney. FEBS Lett. 2000; 473(2): 173-6.

33. Eshraghi-Jazi F, Talebi A, Ashrafi F. Gender difference in Cisplatin-induced nephrotoxicity in a rat model: greater intensity of damage in male than female. Nephrourol Mon. 2013; 5: 818-21.

34. Joseph S, Nicolson TJ, Hammons G, et al. Expression of drug transporters in human kidney: impact of sex, age, and ethnicity. Biol Sex Differ. 2015; 6:4.

35. Scott LA, Madan E, Valentovic MA. Attenuation of cisplatin nephrotoxicity by streptozotocin-induced diabetes. Fundam Appl Toxicol. 1989; 12(3):530-9.

36. Scott LA, Madan E, Valentovic MA. Influence of streptozotocin (STZ)-induced diabetes, dextrose diuresis and acetone on cisplatin nephrotoxicity in Fischer 344 (F344) rats. Toxicology. 1990; 60(1-2): 109-25.

37. da Silva Faria MC, Santos NA, Carvalho Rodrigues MA, et al. Effect of diabetes on biodistribution, nephrotoxicity and antitumor activity of cisplatin in mice. Chem Biol Interact. 2015; 229: 119-31. 